\title{
Competição de clones de mandioquinha-salsa em quatro épocas de colheita
}

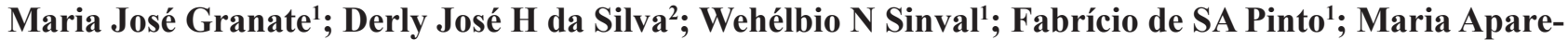 \\ cida N Sediyama ${ }^{1}$; Mário Puiatti ${ }^{2}$ \\ 'EPAMIG-Centro Tecnológico da Zona da Mata, Vila Gianetti, 46, Campus da UFV, 36570-000 Viçosa-MG; ${ }^{2}$ UFV-Dep ${ }^{\text {too. }}$. Fitotecnia, \\ 36570-000 Viçosa-MG; mariajgranate@ufv.br
}

\begin{abstract}
RESUMO
A redução do tempo de permanência no campo da mandioquinhasalsa é um dos principais objetivos do melhoramento. Foi avaliada a produção de 11 clones além de uma cultivar, colhidos aos 187, 243, 306 e 370 dias após o plantio, em Araponga-MG. Os tratamentos foram arranjados como fatorial $12 \times 4$, no delineamento de blocos casualizados, com três repetições. Avaliou-se a altura e diâmetro de planta, comprimento da maior raiz, diâmetro da raiz mais longa, diâmetro da raiz mais grossa, produtividade de rebentos, número de rebentos/planta, produtividade da coroa, produtividade da parte aérea e produtividade de raízes comercializáveis e não-comercializáveis. Estimou-se o tempo que cada clone necessita para atingir produtividade de raízes comercializáveis igual à média de Minas Gerais $(11 \mathrm{t}$ ha $^{-1}$ ). O tempo variou de 243 a 344 dias, sendo que todos os clones tiveram tempo estimado inferior aos 365 dias necessários em campos tradicionais da região. O clone BGH 5742 atingiu 13,46 tha ${ }^{-1}$ aos 243 DAP e o menor tempo estimado para atingir a produtividade média de Minas Gerais. Os clones BGH 4550, BGH 5742, BGH 5746, BGH 5747, BGH 6417, BGH 6507, BGH 6521 e BGH 7607 produziram acima da média de Minas Gerais, aos 306 DAP. As correlações genotípicas da característica produtividade de raízes comercializáveis com as outras características foram baixas ou nulas. As correlações ambientais superaram as genotípicas o que evidencia forte influência do ambiente sobre as plantas.
\end{abstract}

Palavras-chave: Arracacia xanthorrhiza, batata-baroa, precocidade, produtividade, raízes, correlação de caracteres.

\begin{abstract}
Arracacha clones competition at four harvest times

A major objective of arracacha breeding programs is to reduce the crop permanence in field. The yield of 11 clones and one cultivar of arracacha was evaluated at 187, 243, 306 and 370 days after planting in Araponga, Minas Gerais State, Brazil. The treatments were arranged in a factorial $12 \times 4$ scheme in randomized blocks design with three replications. Plant height, plant diameter, length of the longest root, diameter of the longest root, diameter of the thickest root, shoot yield, shoot number per plant, crown yield, aerial part yield, yield of marketable roots and yield of non-marketable roots were evaluated. The time each clone took to reach the average yield of Minas Gerais State (11 tha-1) varied from 243 to 344 days and it was inferior to 365 days, the usual arracacha cycle in that region, for all the evaluated clones.. The clone BGH 5742 produced 13,46 t ha-1 after 243 days in field and presented the shortest estimated time to reach the Minas Gerais State average yield. The clones BGH 4550, BGH 5742, BGH 5746, BGH 5747, BGH 6417, BGH 6507, BGH 6521 and BGH 7607 produced less than the Minas Gerais State average yield after 306 days in field. Genotypic correlations of marketable root yield with other traits were low or null. Environmental correlations overcame the genotypic ones, and it evidenced a strong environmental influence on those plants.
\end{abstract}

Keywords: Arracacia xanthorrhiza, peruvian carrot, earliness, yield, roots, trait correlations.

(Recebido para publicação em 8 de janeiro de 2009; aceito em 1 de dezembro de 2009)

(Received in January 8, 2009; accepted in December 1, 2009)

$\mathrm{T}$ radicionalmente, considera-se que a mandioquinha-salsa necessita de 365 dias de ciclo e pode permanecer mais 60 dias no campo para aguardar melhor preço (Sediyama et al., 2005).

A produção de raízes comercializáveis aos 240 a 300 dias após o plantio (DAP) não é viável a não ser que se utilizem tecnologias de produção ou melhoramento genético (Câmara, 1984), obtendo-se então produtividade superior à média de MG (Granate et al., 2004) e aos 365 dias rendimento superior a 25,0 t ha $^{-1}$ (Santos, 1997). Em Dourados-MS, é economicamente viável a colheita dos 6,5 meses aos 8-9 meses para determinados genótipos (Vieira, 1995) e em Lavras-MG, a colheita obtida aos 305 dias de ciclo foi considerada rentável, embora realizada antes de ser atingido o máximo da produção (Madeira et al., 2000).

Os ciclos de mandioquinha-salsa empregados por diversos pesquisadores são variáveis, mas não têm sido objeto de pesquisas. Para estudos de fertilização, ciclo de 240 dias foi utilizado por Vieira et al. (1996) em Dourados, de 10 meses por Mesquita Filho et al. (1996) em Brasília, de 360 dias por Portz et al. (2003) e por Portz et al. (2006) em Nova Friburgo-RJ. Para estudo de características de mudas um ciclo de nove meses foi usado por Vieira et al. (1998) em Dourados.

Em trabalho sobre desempenho de 
clones um ciclo de 305 dias foi empregado por Madeira et al. (2002) em LavrasMG e em trabalho de consorciação com alface e beterraba um ciclo de 222 dias foi utilizado por Tolentino Júnior et al. (2002) em Dourados.

$\mathrm{Na}$ Zona da Mata (MG), as duas épocas de plantio que favorecem o desenvolvimento das plantas são a de março-junho e a de setembro-outubro, o que evita extremos de temperaturas e de umidade na fase de implantação do ciclo (Granate et al., 2006). Neste caso, as respectivas colheitas são realizadas de janeiro a junho e de julho a outubro do ano seguinte, mantendo-se a cultura no campo de 300 a 365 DAP.

A cotação das raízes na CEASA (MG) atinge os maiores valores entre janeiro e abril e os menores entre julho e outubro (Sediyama et al., 2005). Os produtores da Zona da Mata (MG) preferem a época de plantio de janeiro a abril porque favorece o desenvolvimento da cultura e simultaneamente permite obtenção de melhor preço.

Se for conhecida a menor duração do ciclo, as colheitas podem ser programadas para o período de melhor preço, efetuando-se colheitas semanais, a partir do momento em que as raízes atingirem o tamanho comercializável (Sediyama et al., 2005). A redução do tempo de permanência da cultura no campo é um dos objetivos do melhoramento desta cultura para melhorar o rendimento do produtor (Bustamante et al., 1997; Izquierdo \& Roca, 1998) e uma das demandas dos produtores da região da Zona da Mata (MG).

Neste trabalho, compararam-se as produtividades de raízes de mandioquinha-salsa obtidas aos 187, 243, 306 e 370 DAP para determinar o menor período de tempo que clones produtivos, plantados na mesma época, necessitam permanecer no campo para obtenção de raízes comercializáveis, em Araponga (MG).

\section{MATERIAL E MÉTODOS}

O experimento foi conduzido em propriedade de produtor de mandioquinha-salsa localizada no município de Araponga, de coordenadas geográficas $20^{\circ} 40^{\prime}$ latitude Sul e $42^{\circ} 31^{\prime}$ 'longitude Oeste, de altitude média $1.000 \mathrm{~m}$, e em solo Podzólico Vermelho Amarelo Câmbico Fase Terraço. Foram avaliados os clones BGH 4550, BGH 5742, BGH 5744, BGH 5746, BGH 5747 , BGH 6417, BGH 6425, BGH 6507, BGH 6521, BGH 6525 e BGH 7607, selecionados como produtivos e sem sintomas de doenças na época da colheita em experimento anterior (Granate et al., 2004) e com a cultivar comercial 'Amarela de Senador Amaral' como testemunha. Foram realizadas colheitas aos 187, 243, 306 e 370 DAP, o que correspondeu aos meses de setembro e novembro de 2003 e janeiro e março de 2004, respectivamente.

$\mathrm{O}$ delineamento foi em blocos ao acaso, no esquema fatorial $12 \times 4$ (clones $\mathrm{x}$ épocas de colheita), com três repetições. Cada parcela foi constituída de três linhas contendo oito plantas cada, sendo avaliadas duas plantas competitivas por parcela.

Antes da aração, aplicou-se calcário dolomítico em toda a área, incorporado na camada de 0 a $20 \mathrm{~cm}$ de profundidade. Na época do plantio, aplicaram-se $88 \mathrm{~g} \mathrm{~m}^{-1}$ de sulco da mistura de $15 \mathrm{~kg}$ de cloreto de potássio com $149 \mathrm{~kg}$ de superfosfato simples granulado, conforme resultado da análise de solo.

No terreno, após arado, foram levantadas leiras com $30 \mathrm{~cm}$ de altura, espaçadas de $80 \mathrm{~cm}$; sobre as leiras foram abertos pequenos sulcos onde se plantaram as mudas espaçadas de $40 \mathrm{~cm}$.

Utilizou-se como muda a parte apical do rebento, com cerca de $3 \mathrm{~cm}$ de comprimento, tendo a base cortada em bisel simples e o ápice também cortado, mantendo-se cerca de $1 \mathrm{~cm}$ de pecíolo acima da gema apical conforme Vieira et al. (1996). O plantio foi feito em 19 de março de 2003.

A irrigação foi feita sempre que se considerou necessário quando da ausência de precipitação, por aspersão convencional.

Foram feitas capinas manuais com enxadas para manter a cultura sem competição de ervas daninhas até à cobertura total do terreno pela cultura o que ocorreu aos três meses após o plantio. Não ocorreram surtos de pragas ou doenças.

Foram avaliadas as características: sobrevivência, produtividade de raízes comercializáveis em $\mathrm{t} \mathrm{ha}{ }^{-1}$; altura de planta (média da altura de duas plantas competitivas tomadas ao acaso na parcela, medida do solo até à ponta da folha mais alta em $\mathrm{cm}$ ); diâmetro de planta (média do diâmetro de duas plantas competitivas tomadas ao acaso na parcela, medido passando pelo centro da planta em $\mathrm{cm}$ ); comprimento da maior raiz em cm; diâmetro da raiz mais longa em cm; diâmetro da raiz mais grossa em cm; produtividade dos rebentos em $\mathrm{t} \mathrm{ha}^{-1}$; número de rebentos por planta; produtividade da coroa em $\mathrm{t}$ $\mathrm{ha}^{-1}$; produtividade da parte aérea em $\mathrm{t}$ $\mathrm{ha}^{-1}$; produtividade de raízes comercializáveis e não-comercializáveis (refugo) em $\mathrm{tha}^{-1}$.

As análises de variância e de regressão de modelos lineares simples e quadrático da produtividade em função dos dias após o plantio, os testes de normalidade de Lilliefors e de homogeneidade de variâncias de Bartllet foram realizados. As médias dos tratamentos foram comparadas entre si usando o teste de Tukey a 5\% de probabilidade. As correlações genotípicas e ambientais foram estimadas. O Programa Genes (Cruz, 2001) foi utilizado em todas as análises estatísticas.

\section{RESULTADOS E DISCUSSÃO}

Ocorreram em média 13\% de falhas na emergência das parcelas o que é freqüente nessa cultura.

A altura de planta apresentou valores e variação semelhantes aos observados por Soares (1991), Vieira (1996) e Vieira et al. (1998) e conforme o hábito de crescimento da espécie, com máxima para o clone BGH 5746 aos 243 DAP.

Nas quatro épocas de colheita, a correlação genotípica entre altura de planta e produtividade de raízes comercializáveis é considerada nula e fracamente influenciada pelo ambiente porque a correlação ambiental foi de baixo valor, confirmando a observação de Madeira et al. (2002) de que a altura da planta não é uma característica adequada para 
Tabela 1. Altura de plantas (AP), diâmetro de plantas (DP), comprimento da maior raiz (CMR), diâmetro da raiz mais longa (DMR), diâmetro da raiz mais grossa (DMax), produtividade de rebentos (PReb), número de rebentos por planta (NReb), produtividade da coroa (PCor), produtividade da parte aérea (PPA), produtividade de raízes não-comercializáveis (PRef), de mandioquinha-salsa, em quatro épocas de colheita (plant height (AP), plant diameter (DP), length of the longest root (CMR), diameter of the longest root (CMR), diameter of the thickest root (DMax), shoots yield (PReb), shoots number/plant (NReb), crown yield (PCor), aerial part yield (PPA), yield of non-marketable roots (PRef) of arracacha at four harvest times). Araponga, EPAMIG, 2003-2004.

\begin{tabular}{|c|c|c|c|c|c|c|c|c|c|c|}
\hline Médias ${ }^{1}$ & $\begin{array}{c}\text { AP } \\
(\mathrm{cm})\end{array}$ & $\begin{array}{c}\text { DP } \\
(\mathrm{cm})\end{array}$ & $\begin{array}{c}\text { CMR } \\
(\mathrm{cm})\end{array}$ & $\begin{array}{l}\text { DMR } \\
(\mathrm{cm})\end{array}$ & $\begin{array}{c}\text { DMax } \\
\text { (cm) }\end{array}$ & $\begin{array}{c}\text { PReb } \\
\left(\mathbf{t ~ h a}^{-1}\right)\end{array}$ & $\begin{array}{c}\text { NReb } \\
\text { (un) }\end{array}$ & $\begin{array}{c}\text { PCor } \\
\left(\mathbf{t ~ h a}^{-1}\right)\end{array}$ & $\begin{array}{c}\text { PPA } \\
\left(\mathbf{t ~ h a}^{-1}\right)\end{array}$ & $\begin{array}{c}\text { PRef } \\
\left(\mathrm{t} \mathrm{ha}^{-1}\right)\end{array}$ \\
\hline & \multicolumn{10}{|c|}{187 dias após o plantio } \\
\hline 4550 & $31,3 \mathrm{a}$ & $45,83 \mathrm{a}$ & $9,00 \mathrm{a}$ & $1,63 \mathrm{a}$ & $1,73 \mathrm{a}$ & $6,64 \mathrm{ab}$ & $7,00 \mathrm{ab}$ & $1,41 \mathrm{a}$ & $8,05 \mathrm{ab}$ & $0,36 \mathrm{a}$ \\
\hline 5742 & $21,67 \mathrm{a}$ & $46,33 \mathrm{a}$ & $13,67 \mathrm{a}$ & $2,63 \mathrm{a}$ & $2,67 \mathrm{a}$ & $12,89 \mathrm{ab}$ & 9,17 a & $1,74 \mathrm{a}$ & $14,63 \mathrm{ab}$ & $0,99 \mathrm{a}$ \\
\hline 5744 & $27,83 \mathrm{a}$ & $45,17 \mathrm{a}$ & $7,67 \mathrm{a}$ & $1,6 \mathrm{a}$ & $0,8 \mathrm{a}$ & 14,71 a & $9,5 \mathrm{a}$ & $1,33 \mathrm{a}$ & $16,04 \mathrm{a}$ & $1,20 \mathrm{a}$ \\
\hline 5746 & $22,17 \mathrm{a}$ & $42,67 \mathrm{a}$ & $2,33 \mathrm{a}$ & $0,83 \mathrm{a}$ & $0,83 \mathrm{a}$ & $5,03 \mathrm{~b}$ & $5,67 \mathrm{ab}$ & $0,70 \mathrm{a}$ & $5,73 \mathrm{ab}$ & $0,6 \mathrm{a}$ \\
\hline 5747 & $17,00 \mathrm{a}$ & $36,5 \mathrm{a}$ & $6,87 \mathrm{a}$ & $1,53 \mathrm{a}$ & $1,8 \mathrm{a}$ & $6,2 \mathrm{ab}$ & $5,5 \mathrm{ab}$ & $1,17 \mathrm{a}$ & $7,37 \mathrm{ab}$ & $0,68 \mathrm{a}$ \\
\hline 6417 & $14,5 \mathrm{a}$ & 29,83 a & $5,67 \mathrm{a}$ & $0,63 \mathrm{a}$ & $0,63 \mathrm{a}$ & $4,12 \mathrm{~b}$ & $3,17 \mathrm{~b}$ & $0,55 \mathrm{a}$ & $4,66 \mathrm{~b}$ & $0,21 \mathrm{a}$ \\
\hline 6425 & $19,67 \mathrm{a}$ & $42,83 \mathrm{a}$ & $3,33 \mathrm{a}$ & $1,00 \mathrm{a}$ & $1,00 \mathrm{a}$ & $6,9 \mathrm{ab}$ & $6,00 \mathrm{ab}$ & $0,86 \mathrm{a}$ & $7,76 \mathrm{ab}$ & $0,57 \mathrm{a}$ \\
\hline 6507 & $19,0 \mathrm{a}$ & $43,33 \mathrm{a}$ & $12,33 \mathrm{a}$ & $2,7 \mathrm{a}$ & $2,87 \mathrm{a}$ & $8,54 \mathrm{ab}$ & $7,83 \mathrm{ab}$ & $1,62 \mathrm{a}$ & $10,16 \mathrm{ab}$ & $0,89 \mathrm{a}$ \\
\hline 6521 & $21,33 \mathrm{a}$ & $41,0 \mathrm{a}$ & $4,27 \mathrm{a}$ & $1,7 \mathrm{a}$ & $1,7 \mathrm{a}$ & $5,26 \mathrm{~b}$ & $4,33 \mathrm{ab}$ & $1,04 \mathrm{a}$ & $6,30 \mathrm{ab}$ & $1,07 \mathrm{a}$ \\
\hline 6525 & 20,67 a & $44,83 \mathrm{a}$ & $7,17 \mathrm{a}$ & $1,47 \mathrm{a}$ & $1,63 \mathrm{a}$ & $7,66 \mathrm{ab}$ & $6,83 \mathrm{ab}$ & $1,35 \mathrm{a}$ & $9,01 \mathrm{ab}$ & $0,73 \mathrm{a}$ \\
\hline 7607 & 19,67 a & $51,0 \mathrm{a}$ & $11,83 \mathrm{a}$ & $2,9 \mathrm{a}$ & $2,93 \mathrm{a}$ & $9,19 \mathrm{ab}$ & $7,17 \mathrm{ab}$ & $1,09 \mathrm{a}$ & $10,29 a b$ & $0,88 \mathrm{a}$ \\
\hline Testem. & $24,5 \mathrm{a}$ & $55,83 \mathrm{a}$ & $13,27 \mathrm{a}$ & $2,4 \mathrm{a}$ & $3,0 \mathrm{a}$ & $11,41 \mathrm{ab}$ & $6,83 \mathrm{ab}$ & $1,93 \mathrm{a}$ & $13,3 \mathrm{ab}$ & $0,99 \mathrm{a}$ \\
\hline \multirow[t]{2}{*}{ CV (\%) } & 24,71 & 20,54 & 71,45 & 69,68 & 71,81 & 38,71 & 29,09 & 42,15 & 37,96 & 44,41 \\
\hline & \multicolumn{10}{|c|}{243 dias após o plantio } \\
\hline 4550 & $24,50 \mathrm{~b}$ & $49,00 \mathrm{ab}$ & $15,87 \mathrm{a}$ & $3,63 \mathrm{a}$ & $4,03 \mathrm{ab}$ & $14,84 \mathrm{ab}$ & $14,00 \mathrm{a}$ & $2,86 \mathrm{a}$ & $17,71 \mathrm{ab}$ & $0,75 \mathrm{a}$ \\
\hline 5742 & $30,50 \mathrm{~b}$ & $59,83 \mathrm{ab}$ & $16,13 \mathrm{a}$ & $3,90 \mathrm{a}$ & $4,53 \mathrm{a}$ & $27,00 \mathrm{ab}$ & $16,50 \mathrm{a}$ & $3,02 \mathrm{a}$ & $30,03 \mathrm{ab}$ & $1,12 \mathrm{a}$ \\
\hline 5744 & $29,67 \mathrm{~b}$ & $62,33 \mathrm{a}$ & $11,77 \mathrm{a}$ & $2,90 \mathrm{a}$ & $3,60 \mathrm{ab}$ & $27,42 \mathrm{ab}$ & $15,67 \mathrm{a}$ & $2,73 \mathrm{a}$ & $30,16 \mathrm{ab}$ & $1,41 \mathrm{a}$ \\
\hline 5746 & $44,25 \mathrm{a}$ & $60,50 \mathrm{ab}$ & $15,35 \mathrm{a}$ & $3,55 \mathrm{a}$ & $3,70 \mathrm{ab}$ & $34,14 \mathrm{a}$ & $17,00 \mathrm{a}$ & $3,87 \mathrm{a}$ & $38,01 \mathrm{a}$ & $0,82 \mathrm{a}$ \\
\hline 5747 & $23,00 \mathrm{~b}$ & $44,83 \mathrm{~b}$ & $13,70 \mathrm{a}$ & $4,20 \mathrm{a}$ & $4,37 \mathrm{ab}$ & $11,64 \mathrm{~b}$ & $11,33 \mathrm{a}$ & $1,85 \mathrm{a}$ & $13,49 \mathrm{~b}$ & $0,49 \mathrm{a}$ \\
\hline 6417 & $25,67 \mathrm{~b}$ & $51,33 \mathrm{ab}$ & $13,97 \mathrm{a}$ & $2,43 \mathrm{a}$ & $2,90 \mathrm{~b}$ & $15,10 \mathrm{ab}$ & $9,33 \mathrm{a}$ & $6,33 \mathrm{a}$ & $21,43 \mathrm{ab}$ & 0,49 a \\
\hline 6425 & $29,00 \mathrm{~b}$ & $56,50 \mathrm{ab}$ & $11,57 \mathrm{a}$ & $3,10 \mathrm{a}$ & $3,47 \mathrm{ab}$ & $22,16 \mathrm{ab}$ & $16,67 \mathrm{a}$ & $2,92 \mathrm{a}$ & $25,08 \mathrm{ab}$ & $1,69 \mathrm{a}$ \\
\hline 6507 & $26,50 \mathrm{~b}$ & $50,50 \mathrm{ab}$ & $12,10 \mathrm{a}$ & $3,45 \mathrm{a}$ & $3,55 \mathrm{ab}$ & $16,56 \mathrm{ab}$ & $12,83 \mathrm{a}$ & $2,73 \mathrm{a}$ & $19,30 \mathrm{ab}$ & $0,74 \mathrm{a}$ \\
\hline 6521 & $26,00 \mathrm{~b}$ & $51,17 \mathrm{ab}$ & $12,47 \mathrm{a}$ & $3,80 \mathrm{a}$ & $4,32 \mathrm{ab}$ & $16,12 \mathrm{ab}$ & $11,17^{\mathrm{a}}$ & $3,57 \mathrm{a}$ & $19,69 a b$ & $0,86 \mathrm{a}$ \\
\hline 6525 & $30,50 \mathrm{~b}$ & $58,83 \mathrm{ab}$ & 14,93 a & $3,73 \mathrm{a}$ & $4,03 \mathrm{ab}$ & $23,33 \mathrm{ab}$ & $13,67 \mathrm{a}$ & $2,24 \mathrm{a}$ & $25,57 \mathrm{ab}$ & $0,52 \mathrm{a}$ \\
\hline 7607 & $28,17 \mathrm{~b}$ & $57,17 \mathrm{ab}$ & $13,97 \mathrm{a}$ & $3,90 \mathrm{a}$ & $4,08 \mathrm{ab}$ & $20,23 \mathrm{ab}$ & $14,50 \mathrm{a}$ & $2,34 \mathrm{a}$ & $22,58 \mathrm{ab}$ & $1,17 \mathrm{a}$ \\
\hline Testem. & $30,00 \mathrm{~b}$ & $55,67 \mathrm{ab}$ & $15,17 \mathrm{a}$ & $4,72 \mathrm{a}$ & $4,82 \mathrm{a}$ & $25,63 \mathrm{ab}$ & $18,50 \mathrm{a}$ & $3,54 \mathrm{a}$ & $29,17 \mathrm{ab}$ & $1,12 \mathrm{a}$ \\
\hline \multirow[t]{2}{*}{ CV (\%) } & 12,48 & 10,5 & 13,69 & 25,64 & 13,09 & 32,19 & 22,68 & 66,72 & 30,17 & 55,29 \\
\hline & \multicolumn{10}{|c|}{306 dias após o plantio } \\
\hline 4550 & $32,17 \mathrm{ab}$ & $58,33 \mathrm{a}$ & $16,67 \mathrm{a}$ & $4,58 \mathrm{a}$ & $4,92 \mathrm{a}$ & $17,64 \mathrm{a}$ & $17,33 \mathrm{a}$ & $3,01 \mathrm{a}$ & $20,65 \mathrm{a}$ & $0,82 \mathrm{a}$ \\
\hline 5742 & $38,25 \mathrm{ab}$ & $64,75 \mathrm{a}$ & $12,67 \mathrm{a}$ & $4,33 \mathrm{a}$ & $5,00 \mathrm{a}$ & $31,65 \mathrm{a}$ & $21,08 \mathrm{a}$ & $5,21 \mathrm{a}$ & $36,86 \mathrm{a}$ & $1,18 \mathrm{a}$ \\
\hline 5744 & $43,58 \mathrm{a}$ & 84,08 a & $16,67 \mathrm{a}$ & $3,98 \mathrm{a}$ & $4,55 \mathrm{a}$ & $41,46 \mathrm{a}$ & $21,83 \mathrm{a}$ & $3,46 \mathrm{a}$ & $44,92 \mathrm{a}$ & $1,08 \mathrm{a}$ \\
\hline 5746 & $37,61 \mathrm{ab}$ & 68,03 a & $14,70 \mathrm{a}$ & $5,37 \mathrm{a}$ & $5,57 \mathrm{a}$ & $40,02 \mathrm{a}$ & 28,78 a & $2,17 \mathrm{a}$ & 42,18 a & $0,57 \mathrm{a}$ \\
\hline 5747 & $33,17 \mathrm{ab}$ & 56,17 a & $13,50 \mathrm{a}$ & $4,46 \mathrm{a}$ & $4,91 \mathrm{a}$ & $16,32 \mathrm{a}$ & $16,00 \mathrm{a}$ & $3,45 \mathrm{a}$ & 19,77 a & $0,81 \mathrm{a}$ \\
\hline 6417 & $38,44 \mathrm{ab}$ & 72,17 a & $16,77 \mathrm{a}$ & $4,68 \mathrm{a}$ & $4,68 \mathrm{a}$ & 32,79 a & $21,25 \mathrm{a}$ & $4,74 \mathrm{a}$ & $37,53 \mathrm{a}$ & $0,96 \mathrm{a}$ \\
\hline 6425 & $31,03 \mathrm{ab}$ & $48,19 \mathrm{a}$ & $14,50 \mathrm{a}$ & $4,20 \mathrm{a}$ & $5,23 \mathrm{a}$ & 27,13 a & $21,94 \mathrm{a}$ & $4,13 \mathrm{a}$ & $31,26 \mathrm{a}$ & $0,91 \mathrm{a}$ \\
\hline 6507 & $37,08 \mathrm{ab}$ & $74,17 \mathrm{a}$ & $12,50 \mathrm{a}$ & $4,40 \mathrm{a}$ & $5,17 \mathrm{a}$ & 29,89 a & $22,83 \mathrm{a}$ & $4,82 \mathrm{a}$ & 34,70 a & $0,72 \mathrm{a}$ \\
\hline 6521 & $25,75 \mathrm{~b}$ & $46,67 \mathrm{a}$ & 16,93 a & $4,50 \mathrm{a}$ & $4,73 \mathrm{a}$ & 14,39 a & 16,67 a & $4,00 \mathrm{a}$ & 18,38 a & $0,60 \mathrm{a}$ \\
\hline 6525 & $33,17 \mathrm{ab}$ & $61,17 \mathrm{a}$ & $13,27 \mathrm{a}$ & $5,17 \mathrm{a}$ & $5,17 \mathrm{a}$ & $24,14 \mathrm{a}$ & $19,33 \mathrm{a}$ & $4,09 \mathrm{a}$ & $28,23 \mathrm{a}$ & $0,56 \mathrm{a}$ \\
\hline 7607 & $37,17 \mathrm{ab}$ & $74,42 \mathrm{a}$ & $13,50 \mathrm{a}$ & $4,70 \mathrm{a}$ & $5,00 \mathrm{a}$ & 31,93 a & $27,67 \mathrm{a}$ & $3,70 \mathrm{a}$ & $35,62 \mathrm{a}$ & $1,05 \mathrm{a}$ \\
\hline Testem. & $29,33 \mathrm{ab}$ & $52,00 \mathrm{a}$ & $13,83 \mathrm{a}$ & $4,70 \mathrm{a}$ & $5,44 \mathrm{a}$ & $16,86 \mathrm{a}$ & $16,92 \mathrm{a}$ & $3,58 \mathrm{a}$ & $20,44 \mathrm{a}$ & $0,43 \mathrm{a}$ \\
\hline \multirow[t]{2}{*}{$\begin{array}{l}\mathrm{CV}(\%) \\
\end{array}$} & 16,58 & 21,55 & 15,18 & 19,88 & 14,6 & 39,66 & 25,6 & 30,37 & 37,26 & 51,12 \\
\hline & \multicolumn{10}{|c|}{370 dias após o plantio } \\
\hline 4550 & $33,67 \mathrm{cde}$ & $57,17 \mathrm{e}$ & $19,67 \mathrm{a}$ & $5,47 \mathrm{a}$ & $5,7 \mathrm{a}$ & $20,72 \mathrm{f}$ & $22,33 \mathrm{~d}$ & $4,66 \mathrm{a}$ & $25,37 \mathrm{~g}$ & $1,03 \mathrm{a}$ \\
\hline 5742 & $37,00 \mathrm{~b}$ & $69,58 \mathrm{bc}$ & $14,57 \mathrm{~cd}$ & $5,43 \mathrm{a}$ & $6,00 \mathrm{a}$ & $37,87 \mathrm{~d}$ & $27,33 \mathrm{c}$ & $6,04 \mathrm{a}$ & $43,91 \mathrm{de}$ & $1,11 \mathrm{a}$ \\
\hline 5744 & $43,42 \mathrm{a}$ & $80,25 \mathrm{a}$ & 15,47 bcd & $4,63 \mathrm{a}$ & $5,00 \mathrm{a}$ & 54,52 a & $31,00 \mathrm{~b}$ & $4,07 \mathrm{a}$ & $58,59 \mathrm{a}$ & $0,81 \mathrm{a}$ \\
\hline 5746 & $35,5 \mathrm{bc}$ & $71,86 \mathrm{~b}$ & $13,27 \mathrm{~d}$ & $3,87 \mathrm{a}$ & $4,6 \mathrm{a}$ & $41,53 \mathrm{c}$ & $38,42 \mathrm{a}$ & $5,39 \mathrm{a}$ & $46,91 \mathrm{c}$ & $0,51 \mathrm{a}$ \\
\hline 5747 & $31,58 \mathrm{e}$ & $53,25 \mathrm{f}$ & $16,3 \mathrm{bc}$ & $4,76 \mathrm{a}$ & $5,3 \mathrm{a}$ & $14,49 \mathrm{~g}$ & $19,00 \mathrm{e}$ & $4,20 \mathrm{a}$ & $18,7 \mathrm{~h}$ & $0,95 \mathrm{a}$ \\
\hline 6417 & 34,00 cde & $70,83 \mathrm{bc}$ & $16,57 \mathrm{bc}$ & $4,97 \mathrm{a}$ & $5,37 \mathrm{a}$ & $37,79 \mathrm{~d}$ & $33,25 \mathrm{~b}$ & $4,74 \mathrm{a}$ & $42,53 \mathrm{e}$ & $0,75 \mathrm{a}$ \\
\hline 6425 & 32,89 cde & $62,11 \mathrm{~d}$ & $14,39 \mathrm{~cd}$ & $4,93 \mathrm{a}$ & $5,59 \mathrm{a}$ & $27,02 \mathrm{e}$ & $27,33 \mathrm{c}$ & $3,29 \mathrm{a}$ & $30,31 \mathrm{f}$ & $0,68 \mathrm{a}$ \\
\hline 6507 & $34,58 \mathrm{bcd}$ & $63,0 \mathrm{~d}$ & $14,27 \mathrm{~cd}$ & $4,77 \mathrm{a}$ & $5,6 \mathrm{a}$ & $26,02 \mathrm{e}$ & $26,67 \mathrm{c}$ & $4,42 \mathrm{a}$ & $30,44 \mathrm{f}$ & $1,05 \mathrm{a}$ \\
\hline 6521 & $32,25 \mathrm{de}$ & 55,83 ef & $17,56 \mathrm{ab}$ & $5,4 \mathrm{a}$ & $5,83 \mathrm{a}$ & $22,72 \mathrm{f}$ & $22,58 \mathrm{~d}$ & $3,41 \mathrm{a}$ & $26,14 \mathrm{~g}$ & $0,74 \mathrm{a}$ \\
\hline 6525 & $34,67 \mathrm{bcd}$ & $68,67 \mathrm{c}$ & $14,86 \mathrm{bcd}$ & $5,76 \mathrm{a}$ & $6,96 \mathrm{a}$ & $42,07 \mathrm{c}$ & $32,33 \mathrm{~b}$ & $4,52 \mathrm{a}$ & $46,59 \mathrm{~cd}$ & $0,87 \mathrm{a}$ \\
\hline 7607 & $37,42 \mathrm{~b}$ & $70,83 \mathrm{bc}$ & $20,00 \mathrm{a}$ & $6,13 \mathrm{a}$ & $7,03 \mathrm{a}$ & $46,67 \mathrm{~b}$ & $33,5 \mathrm{~b}$ & $6,24 \mathrm{a}$ & $52,90 \mathrm{~b}$ & $1,42 \mathrm{a}$ \\
\hline Testem. & $32,0 \mathrm{de}$ & 54,58 ef & $16,33 \mathrm{bc}$ & $6,03 \mathrm{a}$ & $6,7 \mathrm{a}$ & $22,65 \mathrm{f}$ & $26,66 \mathrm{c}$ & $5,09 \mathrm{a}$ & $27,74 \mathrm{fg}$ & $1,04 \mathrm{a}$ \\
\hline CV (\%) & 12,08 & 11,24 & 16,96 & 22,59 & 18,78 & 28,24 & 25,37 & 29,79 & 27,07 & 54,86 \\
\hline
\end{tabular}

${ }^{1}$ Médias seguidas pela mesma letra, nas linhas, não diferem estatisticamente entre si, pelo teste de Tukey, a 5\% de probabilidade (means followed by the same letter in lines are not different according to Tukey test at $5 \%$ probability). 
a seleção de clones produtivos, mas ao contrário de Vieira et al. (1998) que obtiveram correlações genotípicas altas, ambientais baixas e consideraram que o bom desenvolvimento da parte aérea permite prognosticar boa produção de raízes (Tabelas 1 e 2 ).

O diâmetro de planta, com máximos do clone BGH 5744 nas três últimas colheitas também apresentou correlações

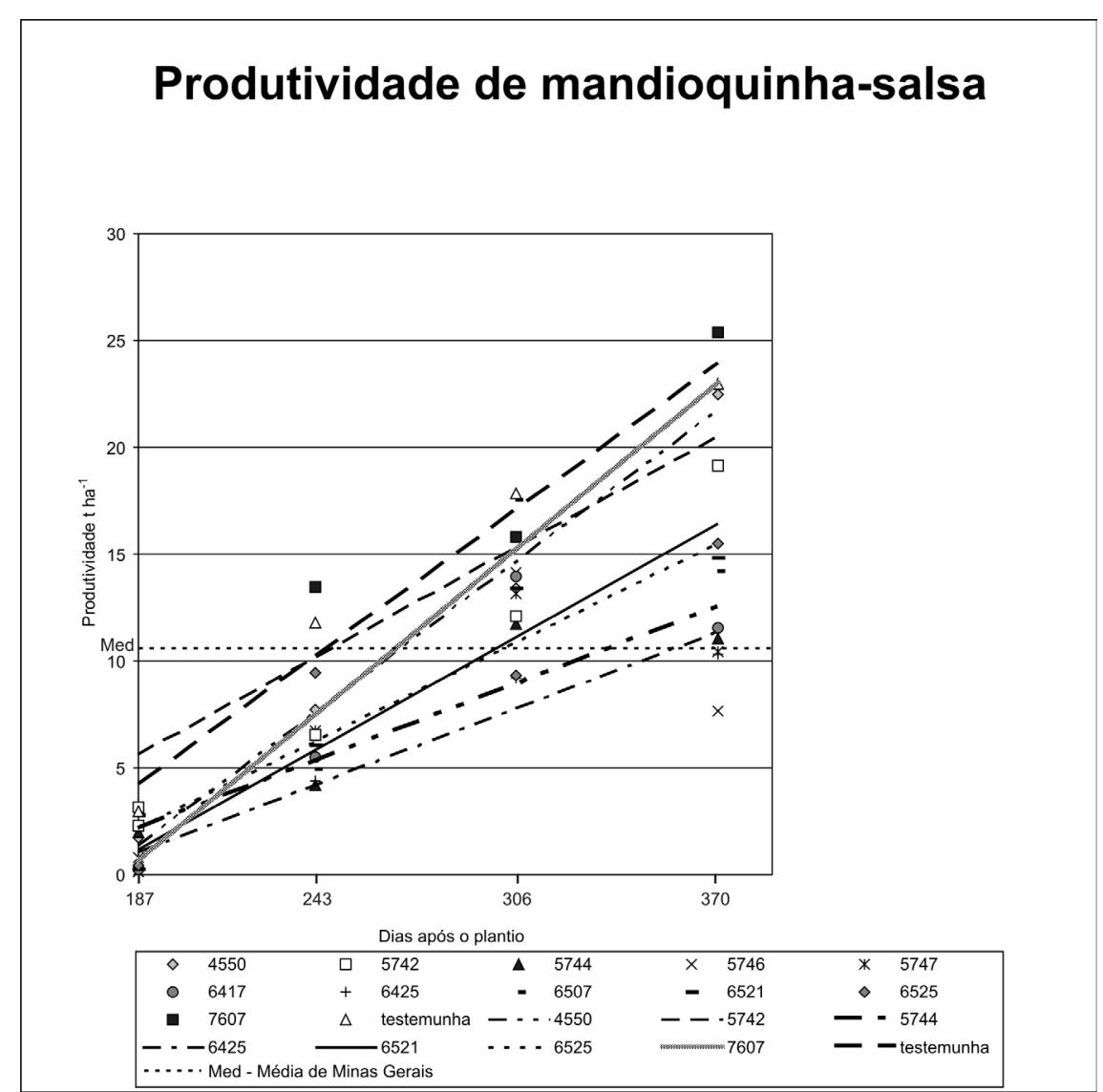

Figura 1. Produtividade $\left(\mathrm{t} \mathrm{ha}^{-1}\right)$ de raízes comercializáveis de mandioquinha-salsa em função dos dias após o plantio (Yield $\left(\mathrm{t} \mathrm{ha}^{-1}\right)$ of marketable roots of arracacha as function of days after planting). Araponga, EPAMIG, 2003-2004.

Tabela 2. Equações de regressão da produtividade em função dos dias após o plantio (equations of regressions of yield as a function of days after planting). Araponga, EPAMIG, 2003-2004.

\begin{tabular}{lll}
\hline Clones BGH & Equação $^{\mathbf{1}}$ & $\mathbf{R}^{\mathbf{2}}$ \\
\hline 4550 & $\hat{Y}=-19,39+0,11 * * x$ & 0,99 \\
5742 & $\hat{Y}=-9,59+0,08 * x$ & 0,87 \\
5744 & $\hat{Y}=-8,40+0,06 * x$ & 0,84 \\
5746 & $\hat{Y}=n s$ & 0,43 \\
5747 & $\hat{Y}=n s$ & 0,71 \\
6417 & $\hat{Y}=n s$ & 0,77 \\
6425 & $\hat{Y}=-9,42+0,06 * x$ & 0,94 \\
6507 & $\hat{Y}=n s$ & 0,71 \\
6521 & $\hat{Y}=-14,35+0,08 * x$ & 0,94 \\
6525 & $\hat{Y}=-11,48+0,07 * x$ & 0,85 \\
7607 & $\hat{Y}=-22,39+0,12 * x$ & 0,94 \\
Testemunha & $\hat{Y}=-15,85+0,10 x$ & 0,98 \\
\hline
\end{tabular}

$1 ; * * * *$ : significativos a 1 e $5 \%$ de probabilidade pelo teste $\mathrm{F}$ ( $\mathrm{F}$ test significant at 1 and $5 \%$ of probability); ns: teste $\mathrm{F}$ não significativo (non significant $\mathrm{F}$ test). genotípicas próximas de zero com produtividade de raízes comercializáveis e sem forte influência ambiental. A falta de correlações genotípicas e ambientais deverá permitir adensamento da cultura e conseqüente aumento da produtividade (Madeira et al., 2002) (Tabelas 1 e 2).

O comprimento da maior raiz (CMR), diâmetro da raiz mais longa (DMR) e diâmetro da raiz mais grossa (DMax) não apresentaram diferenças estatísticas entre clones com exceção de DMax aos 243 DAP e CMR aos 370 DAP (Tabela 2). O DMax foi a característica mais consistente para avaliação das propriedades físicas da planta, por isso deverá ser sempre considerado em avaliações visando o melhoramento (Kimura \& Ayub, 1995). No entanto, neste trabalho, as suas correlações genotípicas e ambientais com produtividade de raízes comercializáveis foram inferiores a 0,80 (Tabelas 1 e 2).

A produtividade de rebentos foi muito superior, nas quatro colheitas, à de massa fresca de rebentos, de 3,92 $\mathrm{t} \mathrm{ha}^{-1}$ aos 222 dias após o plantio, observada por Tolentino Júnior et al. (2002). A sua correlação genotípica com a produtividade de raízes comercializáveis foi muito variável entre colheitas, daí não ser uma característica confiável e utilizável na seleção indireta para produtividade (Tabelas 1 e 2).

O clone Amarelo de Carandaí ou BGH 5746 avaliado por Tolentino Júnior et al. (2002) em Dourados-MS foi também avaliado neste trabalho no qual o número de rebentos por planta, a partir dos 243 DAP, foi muito superior aos 9,38 rebentos por planta observados por Tolentino Júnior et al. (2002). A produtividade de raízes aos 243 DAP deste clone, neste trabalho, foi inferior à de 11,92 $\mathrm{t} \mathrm{ha}^{-1}$ observada por aqueles autores, assim como a produtividade da parte aérea, para a qual Tolentino Júnior et al. (2002) obtiveram 4,36 $\mathrm{t} \mathrm{ha}^{-1}$. A produtividade de rebentos foi superior às 3,92 $\mathrm{t} \mathrm{ha}^{-1}$ obtidas pelos referidos autores, a partir dos 187 DAP. As correlações genotípicas do número de rebentos por planta e produtividade da parte aérea com produtividade de raízes comercializáveis foram muito variáveis entre colheitas, o que não permite utilizá-la na seleção indireta para produtividade 
Tabela 3. Correlações lineares genotípicas e ambientais de várias características com a característica produtividade de raízes comercializáveis de mandioquinha-salsa, em quatro épocas de colheita (linear genotypic and environmental correlations of several traits with trait marketable roots yield of arracacha at four harvest times). Araponga, EPAMIG, 2003-2004.

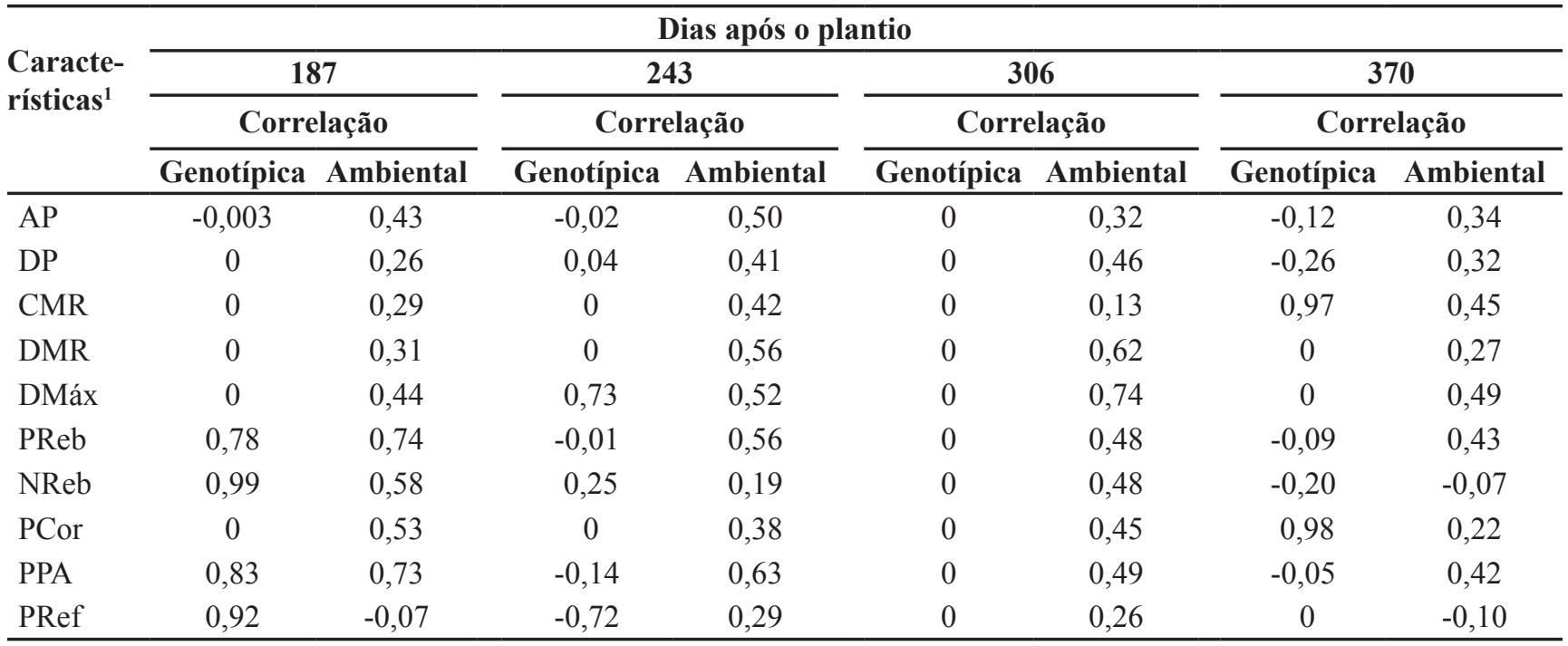

${ }^{1}$ AP: Altura de plantas; DP: diâmetro de plantas; CMR: comprimento da maior raiz; DMR: diâmetro da raiz mais longa; DMax: diâmetro da raiz mais grossa; PReb: produtividade de rebentos; NReb: número de rebentos por planta; PCor: produtividade da coroa; PPA: produtividade da parte aérea; PRef: produtividade de raízes não-comercializáveis (AP: plant height; DP: plant diameter; CMR: length of the longest root; DMR: diameter of the longest root; DMax: diameter of the thickest root; PReb: shoots yield ; NReb: shoots number/plant; PCor: crown yield; PPA: aerial part yield; PRef: yield of non-marketable roots).

(Tabelas 1, 2 e 3).

A produtividade de raízes nãocomercializáveis foi baixa em todas as colheitas e para todos os clones, sem correlações genotípicas de interesse com produtividade (Tabelas 1 e 2).

A produtividade de raízes comercializáveis de todos os clones, na colheita aos 187 DAP, foi inferior à média de MG, de 10,95 tha $\mathrm{t}^{-1}$ (Torres, 1997). Aos 243 DAP o clone BGH 5742 foi muito superior à média de MG. Aos $306 \mathrm{DAP}$ os clones BGH 4550, BGH 5742, BGH 5746, BGH 5747, BGH 6417, BGH 6507, BGH 6521 e BGH 7607 produziram acima da média de MG.

A produtividade média dos 11 clones aos 306 DAP foi superior às obtidas por Portz et al. (2003) e Portz et al. (2006) de $12 \mathrm{t} \mathrm{ha}^{-1}$ aos 300 dias após o transplantio mais 60 dias de viveiro. Aos 370 DAP o clone BGH 7607 e a testemunha foram superiores em mais do dobro à média de $\mathrm{MG}$, enquanto os clones BGH 4550, BGH 5742, BGH 5744, BGH 6417, BGH 6507, BGH 6521, BGH 6525 e BGH 7607 foram superiores à média de MG (Tabela 3).

Todos os clones avaliados atingiriam a média de produtividade de $\mathrm{MG}$, de 11 t ha ${ }^{-1}$ antes dos 12 meses após o plantio, tradicionais na cultura (Figura 1). O valor de produção máxima estimado por Mesquita Filho et al. (1996) foi de 12,9 t ha $^{-1}$, em Brasília, aos 270 DAP. O maior acúmulo de fotoassimilados nas raízes de reserva verificou-se aos 300 DAT (transplantio) e a produtividade média do clone Amarelo de Carandaí foi de 12 t ha-1 (Portz et al., 2006).

Os clones avaliados neste trabalho podem ser considerados mais precocemente produtivos, no ambiente de Araponga.

Em Dourados foi obtida a produtividade média de 10 t ha $^{-1}$ aos 270 DAP por Vieira et al. (1998) e considerada viável para aquele estado. Comportamento linear foi observado também por Vieira et al. (1998), Portz et al. (2003) e Portz et al. (2006).

A falta de variabilidade genética entre os clones, atribuída à seleção prévia para a obtenção dos clones deste experimento (Granate et al., 2004), teve por conseqüência estimativas de variâncias genotípicas nulas para várias características e daí as correlações genotípicas também serem nulas.

A grande influência dos fatores am- bientais sobre essa cultura, talvez mais importante que a dos fatores genéticos, é evidenciada pelos resultados das correlações ambientais que superaram as genotípicas (Tabela 2).

A influência do ambiente não permite aconselhar a total substituição dos clones tradicionalmente usados na região, BGH 5746 e BGH 7607, pelos clones que apresentaram melhor desempenho neste trabalho porque esse desempenho pode não se repetir em outros anos.

Considerando que é conveniente a diversidade genética para diminuir os prejuízos de possíveis futuras epifitias (Madeira et al., 2002) e que o clone Araponga ou BGH 7607 é o mais utilizado no município de Araponga, a esse clone poderá ser associado, para colheita aos 243 DAP, o clone BGH 5742 e, para colheita aos 306 DAP, o clone BGH 6507. Essa estratégia deverá permitir bom retorno ao produtor devido à produtividade elevada e à menor permanência da cultura no campo.

A colheita da mandioquinha-salsa aos 180 DAP não é aconselhável considerando a baixa produtividade. Colheitas a partir dos 300 DAP são possíveis visto que aos 306 DAP nove clones ultrapas- 
saram a produtividade média de MG. O tempo estimado para atingir a média de produtividade de MG foi inferior a 365 dias para todos os clones.

Os CV foram compatíveis com os obtidos para mandioquinha-salsa por Vieira (1995), e por Câmara (1984) (Tabela 1). Nas culturas de órgãos subterrâneos como cenoura, mandioca, mandioquinha-salsa e inhame os valores do CV são altos e em geral superiores a 30\% (Soares, 1991).

Os caracteres quantitativos, que em geral controlam as características de importância econômica, são muito influenciados pelos ambientes temporais e espaciais. As interações exercem grande influência sobre esses caracteres e sobre o resultado final das culturas (Allard, 1971). Em conseqüência, as avaliações dos materiais são dificultadas bem como a recomendação para cada região (Falconer, 1987), porque a estrutura genética das populações é mascarada (Torres, 1988).

O teste de normalidade de Lilliefors foi não significativo, indicando que é razoável estudar os dados por meio da distribuição normal. O teste de homogeneidade das variâncias de Bartllet também não foi significativo pelo que não se rejeita a hipótese zero de igualdade das variâncias.

\section{AGRADECIMENTOS}

À FAPEMIG pelo financiamento do Projeto CAG 12/01 "Melhoramento da batata-baroa (Arracacia xanthorrhiza Banc.) para o Estado de Minas Gerais - recomendação de cultivares e seleção populacional" que permitiu a obtenção destes dados e pela Bolsa BAT concedida a Fabrício de Sales Alves Pinto e a Wehélbio Nepomuceno Sinval. À Embrapa pelo fornecimento de mudas da cultivar Amarela de Senador Amaral.

\section{REFERÊNCIAS}

ALLARD RW. 1971. Princípios do melhoramento genético das plantas. Rio de Janeiro: Edgar Blücher, USAID. 381p.

BUSTAMANTEPG; CASALIVWD; SEDIYAMA MAN. 1997. Melhoramento genético da batatabaroa. Informe Agropecuário 19: 16-18.

CÂMARA FLA. 1984. Estudo de tecnologias objetivando precocidade de produção de batata-baroa (Arracacia xanthorrhiza Bancroft) Viçosa: UFV. 54p (Tese mestrado).

CRUZ CD. 2001. Programa Genes versão windows aplicativo computacional em Genética e Estatística. Viçosa: Editora UFV. 648p.

FALCONER DS. 1987. Introdução à genética quantitativa. Viçosa: UFV. 279 p.

GRANATE MJ; SEDIYAMA MAN; PUIATTI M. 2006. Batata-baroa ou mandioquinha salsa (Arracacia xanthorrhiza Banc.). In: PAULA JUNIOR TJ; VENZON M (eds). 101 Culturas: manual de tecnologias agrícolas. Belo Horizonte: EPAMIG. p. 137-142.

GRANATE MJ; SEDIYAMA MAN; OLIVEIRA LR; CRUZ CD; PUIATTI M. 2004. Clonal selection in arracacha breeding. Crop Breeding and Applied Biotechnology 4: 105-110.

IZQUIERDO J; ROCA W. 1998. Under-utilized Andean crops status and prospects of plant biotechnology for the conservation and sustainable agricultural use of genetic resources. Acta Horticulturae 457: 157-172.

KIMURA S; AYUB R. 1995. Determinação de forma e tamanho da batata-baroa (Arracacia xanthorrhiza). Revista Ceres 42: 1-9.

MADEIRA NR; MALUF LE; RESENDE TV. 2000. Avaliação da precocidade de colheita de mandioquinha-salsa em Lavras, MG. Horticultura Brasileira 18: 261.

MESQUITA FILHO MV; SOUZAAF; SANTOS
FF; OLIVEIRA AS. 1996. Resposta da mandioquinha-salsa à adubação com bórax em um latossolo vermelho-escuro distrófico de Cerrado. Horticultura Brasileira 14: 45-48.

PORTZ A; MARTINS CAC; LIMA E. 2003. Crescimento e produção de raízes comercializáveis de mandioquinha-salsa em resposta à aplicação de nutrientes. Horticultura Brasileira 21: 485-488.

PORTZA; MARTINS CAC; LIMA E; ZONTAE. 2006. Teores e acúmulo de nutrientes durante o ciclo da mandioquinha-salsa em função da aplicação de nitrogênio, fósforo e potássio. Horticultura Brasileira 24: 329-333.

SANTOS FF. 1997. Clima, cultivares e época de plantio da mandioquinha-salsa. Informe Agropecuário 19: 35-37.

SEDIYAMA MAN; VIDIGAL SM; GRANATE MJ; SANTOS MR; MASCARENHAS MHT. 2005. Cultura da mandioquinha-salsa ou batata-baroa. Boletim Técnico 77: 28. Belo Horizonte: EPAMIG.

SOARES L. 1991. Melhoramento de batatabaroa (Arracacia xanthorrhiza Banc.) II Divergência genética entre clones com base em procedimentos multivariados e estimativas de parâmetros genéticos. Viçosa: UFV. $75 \mathrm{p}$ (Tese mestrado).

TOLENTINO JÚNIOR CF; ZÁRATE NAH; VIEIRA, MC. 2002. Produção de mandioquinha-salsa consorciada com alface e beterraba. Acta Scientiarum 24: 1447-154.

TORRES G. 1997. Mandioquinha-salsa: alimento energético. Informe Agropecuário 19: 3.

VIEIRA MC. 1995. Avaliação do crescimento e da produção de clones e efeito de resíduo orgânico e fósforo em mandioquinha-salsa no Estado de Mato Grosso do Sul. Viçosa: UFV. 146p (Tese doutorado).

VIEIRA MC; ZÁRATE NAH; SIQUEIRA JG, CASALI VWD. 1996. Crescimento e produção de mandioquinha-salsa em função de características das mudas. Horticultura Brasileira 14: 42-44.

VIEIRA MC; CASALI VWD; CARDOSO AA; MOSQUIM. 1998. Crescimento e produção de mandioquinha-salsa em função da adubação fosfatada e da utilização de cama de aviário. Horticultura Brasileira 16: 68-73. 